\title{
Editorial
}

Adrián LLerena*, Eva Peñas-LLedó, Fernando de Andrés, Carmen Mata-Martín, Cristina L. Sánchez, Agustín Pijierro and Jesús Cobaleda

\section{Clinical implementation of pharmacogenetics and personalized drug prescription based on e-health: the MedeA initiative}

https://doi.org/10.1515/dmpt-2020-0143

Received August 4, 2020; accepted August 5, 2020; published online September 1, 2020

There is a growing demand for the clinical implementation of pharmacogenetics ( $\mathrm{PGx}$ ), personalized and precision medicine (PPM) for drug prescription to reduce adverse drug reactions (ADRs), drug failure, and ultimately health care costs. However, it is convenient to clarify the concept of clinical implementation to realize its benefits. Advances on PGx clinical implementation depend on the integration of genetic along with other relevant biomarkers and clinical information influencing variability in drug response for being interpreted and translated into clinical decisionmaking to optimize drug treatment choice during routine clinical practice.

*Corresponding author: Professor Adrián LLerena MD, PhD, Clinical Pharmacology, INUBE Biosanitary Research Institute of Extremadura, Badajoz, Spain; Faculty of Medicine, University of Extremadura, Badajoz, Spain; CICAB Clinical Research Center - UICEC SCREN CIBERSAM ISCIII, Pharmacogenetics and Personalized Medicine Unit, Badajoz University Hospital, Extremadura Health Service. Badajoz, Spain; and Extremadura University Hospital and Medical School/ Extremadura Health Service. Badajoz, 06080, Extremadura, Spain. Phone: +34 924218040, E-mail: allerena@unex.es

Eva Peñas-LLedó, INUBE Extremadura Biosanitary Research Institute, Badajoz, Spain; Faculty of Medicine, University of Extremadura, Badajoz, Spain

Fernando de Andrés, Carmen Mata-Martín and Cristina L. Sánchez, INUBE Extremadura Biosanitary Research Institute, Badajoz, Spain; CICAB Clinical Research Center, Pharmacogenetics and Personalized Medicine Unit, Badajoz University Hospital, Extremadura Health Service, Badajoz, Spain. https://orcid.org/0000-0003-1076-0743 (F. de Andrés)

Agustín Pijierro, INUBE Extremadura Biosanitary Research Institute, Badajoz, Spain; Department of Internal Medicine, Badajoz University Hospital, Extremadura Health Service, Badajoz, Spain Jesús Cobaleda, INUBE Extremadura Biosanitary Research Institute, Badajoz, Spain; Faculty of Medicine, University of Extremadura, Badajoz, Spain; and Ciudad Jardín Primary Health Care Center, Extremadura Health Service, Badajoz, Spain
There are several initiatives related to $\mathrm{PGx}$ clinical implementation in Europe [1], using either a preventive (pre-emptive) measures approach or a reactive one (point of care) (https://www.icpermed.eu/). Some of them are integrated on different research platforms, that perform the genetic analyses using either single druggene combinations, gene-arrays, or next-generation sequencing (NGS) [1]. Nevertheless, many of them just aim to analyze the relevance of genetic information in the absence of other relevant data influencing drug response variation, without considering polypharmacy in the context of multi-morbidity. PGx applied to pharmacokinetic variability is so far the cornerstone of its clinical implementation [2]. Thus, determining actual phenotypes by analyzing metabolic ratios from actual drug treatments to evaluate enzyme activity (dosedependent phenocopying [3]) as shown during treatment with thioridazine [4], risperidone [5] or fluoxetine [6], including additional influencing factors [7] such as liver or kidney function, and concomitant pharmacological treatments (to prevent drug-drug interactions [8]) becomes essential to develop a precise clinical implementation process.

Therefore, despite the need of applying PPM into daily clinical practice, there are still barriers to overcome regarding the inclusion of all factors relevant for variation in drug response. Moreover, another important limitation is information management. Hence, there is a need for developing computational tools that may integrate the relevance of different factors influencing drug response in a context of polypharmacy to simplify the prescribers' decision-making. Currently, drug selection requires manual data entry, therefore a guided drug prescription e-tool integrated into the Electronic Medical Record (EMR) is needed for improving drug choices in the context of drug poly-therapy and poly-pathology. Moreover, the system needs to be evaluated to establish a cost/effectiveness analysis for its implementation in the Public Health Service. 
"MedeA Initiative" is a PPM strategy (www. proyectomedea.es), which integrates $\mathrm{PGx}$ and other relevant biomarkers (metabolic phenotype), with other relevant information for drug prescription, to develop a DecisionSupporting Tool (Personalized Prescription System or PPS) to be used for individualized drug prescription during regular clinical practice within the context of e-health. The collection of clinical information comes from both the EMR and from wearable sensors for the monitoring of physical and physiological parameters in daily life. The PPS will be implemented in the EMR to allow an individualized drug prescription to all patients. The system will be also accessible by patients using a personalized prescription card. MedeA is being implemented in the Extremadura Health Service (SES), part of the Spanish Health Care System, including a total of 1.1 M inhabitants in both primary and hospital care.

The use of this preventive strategy is expected to contribute to the sustainability of European public health care systems by reducing indirect drug costs caused by the failure of pharmacological therapy and ADRs.

The uniqueness of the MedeA initiative resides on its all-encompassing approach: merging relevant factors that influence variability in drug response in an algorithm that will be integrated into the e-health care system and evaluated afterwards. It will allow a better drug selection for a given patient in the context of all prescribed drugs. The system will benefit not only to personal health care but also to patients, whom will be better informed about their treatment choices.

In conclusion, MedeA will develop a PPS connected to the EMR within the context of e-health care, which will be validated under real clinical conditions. The project aims to be of benefit to patients and clinicians initially from the health care system in Extremadura and later to other similar European health care systems. Clinicians will be assisted by a tailored prescription-supporting tool including genetic and other information from the EMR for selecting the most appropriate drug treatment options, which will be assessed by analytical data with artificial intelligence. Thus, a reduction of drug adverse effects and related burden and costs, as well as people's quality of life improvement is expected.

Research funding: None declared.

Author contributions: All authors have accepted responsibility for the entire content of this manuscript and approved its submission.

Competing interests: Authors state no conflict of interest.

\section{References}

1. Reisberg S, Krebs K, Lepamets M, Kals M, Mägi R, Metsalu K, et al. Translating genotype data of 44,000 biobank participants into clinical pharmacogenetic recommendations: challenges and solutions. Genet Med 2019 Jun;21:1345-54.

2. LLerena A, Peñas-Lledó EM. Metabolic phenotype prediction from genotyping data: a bottleneck for the implementation of pharmacogenetics in drug development and clinical practice. Drug Metab Pers Ther 2015;30:143-5.

3. LLerena A, Berecz R, de la Rubia A, Fernández-Salguero P, Dorado P. Effect of thioridazine dosage on the debrisoquine hydroxylation phenotype in psychiatric patients with different CYP2D6 genotypes. Ther Drug Monit 2001 Dec;23:616-20.

4. Llerena A, Berecz R, de la Rubia A, Norberto MJ, Benítez J. Use of the mesoridazine/thioridazine ratio as a marker for CYP2D6 enzyme activity. Ther Drug Monit 2000;22:397-401.

5. Berecz R, LLerena A, de la Rubia A, Gómez J, Kellermann M, Dorado P, et al. Relationship between risperidone and 9-hydroxy-risperidone plasma concentrations and CYP2D6 enzyme activity in psychiatric patients. Pharmacopsychiatry 2002 Nov;35:231-4.

6. LLerena A, Dorado P, Berecz R, González AP, Peñas-LLedó EM. Effect of CYP2D6 and CYP2C9 genotypes on fluoxetine and norfluoxetine plasma concentrations during steady-state conditions. Eur J Clin Pharmacol 2004;59:869-73.

7. Magalhães $P$, Alves $G$, Fortuna A, Llerena A, Falcão A. Clinical collaborators of the GnG-PK/PD-AD study. Pharmacogenetics and therapeutic drug monitoring of fluoxetine in a real-world setting: A PK/PD analysis of the influence of (non-)genetic factors. Exp Clin Psychopharmacol 2019. https://doi.org/10.1037/ pha0000334.

8. Berecz R, Dorado P, De La Rubia A, Cáceres MC, Degrell I, LLerena A. The role of cytochrome $\mathrm{P} 450$ enzymes in the metabolism of risperidone and its clinical relevance for drug interactions. Curr Drug Targets 2004;5:573-9. 